FORMATION Formation emploi

Revue française de sciences sociales

107 | juillet-septembre 2009

La formation professionnelle en Amérique latine

\title{
Priorité à la formation scolaire pour le travail au mexique. Tensions et contradictions entre l'Etat, les secteurs professionnels et les étudiants.
}

The priority given in Mexico to school-based training for work. Tensions and contradictions between the state, professional sectors and students

Prioridad de la formación escolar para el trabajo en México. Tensiones y contradicciones entre el Estado, los sectores laborales y los estudiantes. Schulausbildung in Mexiko mit Vorrang für die Arbeitswelt. Spannungen und Widersprüche zwischen Staat, Berufssektoren und Schülern

\section{María de Ibarrola}

Traducteur : Mireille Zangani

\section{(2) OpenEdition}

\section{Édition électronique}

URL : http://journals.openedition.org/formationemploi/1985

DOI : 10.4000/formationemploi.1985

ISSN : 2107-0946

Éditeur

La Documentation française

Édition imprimée

Date de publication : 1 septembre 2009

Pagination : 25-39

ISSN : 0759-6340

\section{Référence électronique}

María de Ibarrola, «Priorité à la formation scolaire pour le travail au mexique. Tensions et contradictions entre l'Etat, les secteurs professionnels et les étudiants. », Formation emploi [En ligne], 107 | juillet-septembre 2009, mis en ligne le 01 septembre 2011, consulté le 30 octobre 2020. URL http://journals.openedition.org/formationemploi/1985; DOI : https://doi.org/10.4000/ formationemploi.1985 


\section{DOSSIER}

\section{Priorité à la formation scolaire pour le travail au Mexique. Tensions et contradictions entre l'État, les secteurs professionnels et les étudiants?}

María de lbarrola*

La structure actuelle de la formation professionnelle résulte des tensions entre les propositions du gouvernement fédéral, les interventions indirectes et limitées des entreprises, le degré de consolidation des institutions scolaires elles-mêmes et les aspirations des élèves.

Un des arguments récurrents dans les débats sur le difficile développement économique du Mexique est celui de la faible et inadéquate formation de sa force de travail. Cependant, tout au long du $20^{\text {e }}$ siècle, la plus grande partie de cette période s'étant déroulée sous le gouvernement fédéral, le pays a déployé un effort considérable pour améliorer la scolarité de la population mexicaine, considérant que la responsabilité de l'organisation, de la conception et de la mise en œuvre de la formation pour le travail revenait au système scolaire. Une seconde voie, que nous n'évoquons pas dans cet article, fait obligation constitutionnelle aux entreprises de dispenser de la

${ }^{1}$ L'article s'appuie sur des travaux de recherches antérieurs de l'auteur, référencés en bibliographie. formation professionnelle à leurs travailleurs (Loi fédérale du travail, 153-A à 153-X).

L'intérêt de cet essai est de postuler que la structure actuelle de la formation scolaire pour le travail traduit l'existence de tensions et de contradictions dans la façon dont le gouvernement fédéral a assumé cette responsabilité, la vision sommaire des secteurs professionnels, les intérêts et possibilités des institutions scolaires et les demandes et aspirations des

* Maria de lbarrola est chercheure au département de recherches éducatives, Centre de recherches et d'études avancées de I'Institut polytechnique national (IPN)/ Mexique. Elle remercie Elena Torres pour l'aide apportée dans l'actualisation des statistiques scolaires. 
jeunes, dans un contexte national caractérisé par l'existence de marchés du travail hétérogènes et inégaux (de Ibarrola, 2006). Dans le cadre des très rares recherches sur ce thème dans le pays et de statistiques éparses, notre réflexion s'appuie sur des résultats globaux issus de recherches préalables et offre une première approche de la complexité des interactions qui influent sur l'organisation de la formation de la population active du pays. On constate que l'agenda public a été affecté par l'existence de multiples acteurs aux intérêts divergents, aux rationalités limitées et à l'information insuffisante (Aguilar, 1993) et que les diverses intentions et actions des acteurs produisent des effets collectifs différents (et même pervers) (Boudon, 1980).

Notre thèse s'appuie sur trois angles d'approche. Dans le premier, on analyse le leaderschip du gouvernement fédéral à partir de la moitié du $20^{\text {e }}$ siècle, et la façon dont il a tenté d'intégrer la vision du secteur professionnel, essentiellement des entrepreneurs, aux structures scolaires créées.

Le second angle propose, quant à lui, une approche par la structure hétérogène des marchés du travail du pays et les façons dont s'y expriment les relations entre scolarité, travail et revenus. Le troisième angle analyse la répartition des étudiants entre les niveaux et modalités scolaires, l'expression de leurs intérêts, motivations, et leur degré de connaissance du sens professionnel de la scolarité face aux opportunités scolaires et professionnelles créées. Ces trois angles d'approche s'intègrent dans une dernière partie qui décrit les tensions et contradictions entre les visions des acteurs et renseigne sur les politiques concrètes d'impulsion ou de frein dans certains domaines de formation. Les conclusions orientent vers de nouvelles recherches sur cette trame complexe qui dépasse la vision linéaire et mécaniste de la scolarité comme la solution au problème de travail, de revenus et de développement économique.

\section{LE LEADERSCHIP DU GOUVERNEMENT FÉDÉRAL}

Améliorer la scolarité de la population mexicaine constitua l'un des grands défis auquel fut confronté le pays tout au long du $20^{\text {e }}$ siècle, défi qui s'est accentué en raison de la très faible scolarisation de la population, encore d'actualité au milieu de ce siècle, et par la forte augmentation démographique qui a commencé à se réduire seulement autour des années 80 .

En 1950, le système scolaire accueillait un peu plus de trois millions d'élèves ; en 2006, le chiffre s'élève à plus de trente millions (Solana, 1982, pp. 598-599; SEP, 2008). La population de 3 à 24 ans, quant à elle, passe d'un peu plus de 13 millions en 1950 à un peu plus de 46 millions en 2006 (CONAPO, 2007). L'augmentation des effectifs a été massive : en préscolaire, elle s'élève à 4739234 ; en primaire, à 14585804 ; au secondaire, à 6055467 ; en intermédiaire supérieur, de 37329 étudiants en 1950, elle passe à 3742 943, et dans l'enseignement supérieur, de 29892 à 2582664 (Solana, 1982 ; SEP, 2008). Les opportunités de scolarité pour les enfants et jeunes du pays sont passées de $24 \%$ du groupe d'age à 68,4\% actuellement.

Cependant, nombreux sont les jeunes qui n'ont pas accès à l'école, en particulier à partir de 15 ans. La réponse à la demande des différents groupes d'âge est insuffisante: en 2006, l'effectif des enfants en primaire atteint à peine en moyenne nationale $94,4 \%$ pour le primaire et $93 \%$ pour le secondaire. Mais pour le niveau intermédiaire supérieur, le chiffre chute à 59,7\% du groupe d'âge, et pour le niveau supérieur, il atteint à peine $24,3 \%^{2}$ (SEP - Système pour l'analyse de la statistique éducative). Grâce à tous ces efforts, la scolarisation moyenne de la population active (PEA) de plus de 15 ans du pays est passée de 2,6 ans en 1960 à 8,3 en 2006, inférieure toutefois à la scolarité élémentaire obligatoire pour toute la population qui, en 1993, était de 9 ans et pour 2002 de 12, y compris trois années de préscolaire (Il s'agit des nombreux méxicains qui n'atteignaient même pas l'équivalent du cours élementaire niveau 2 de l'ecole primaire et qui à l'heure actuelle n'atteignent pas le niveau collège).

L'effort pour la scolarisation de la population mexicaine s'est inscrit dans une politique volontaire

\footnotetext{
2 Pour les données de cadrage, nous prendrons comme base les groupes d’âge suivants : primaire : 6-12 ans ; secondaire : 1315 ans ; éducation moyenne supérieure : 16-18 ans ; supérieur, non compris le $3^{\text {e }}$ cycle : 19 à 23 ans.
} 


\section{Encadré 1 \\ Le système éducatif mexicain}

\begin{tabular}{|c|c|c|c|c|c|c|c|}
\hline Âge & Niveau & $\begin{array}{l}\text { Nombre } \\
d^{\prime} \text { années }\end{array}$ & Cycles & \multicolumn{2}{|c|}{ Modalité } & Effectif & $\begin{array}{l}\text { Taux de } \\
\text { couverture }\end{array}$ \\
\hline 1 & & & & \multirow{2}{*}{\multicolumn{2}{|c|}{ Éducation initiale }} & & \\
\hline 2 & & & & & & & \\
\hline 3 & \multirow{3}{*}{ Préscolaire } & \multirow{3}{*}{1 à 3} & \multirow{13}{*}{$\begin{array}{c}\text { Éducation } \\
\text { de base } \\
\text { obligatoire }\end{array}$} & \multicolumn{2}{|c|}{ Générale } & 4205438 & \multirow{3}{*}{73.0} \\
\hline 4 & & & & \multicolumn{2}{|c|}{ Indigène } & 379874 & \\
\hline 5 & & & & \multicolumn{2}{|c|}{ Communautaire } & 153933 & \\
\hline 6 & \multirow{6}{*}{ Primaire } & \multirow{6}{*}{1 à 6} & & \multirow{3}{*}{\multicolumn{2}{|c|}{ Générale }} & & \multirow{6}{*}{$\left.110.0{ }^{*}\right)$} \\
\hline 7 & & & & & & $\begin{array}{c}1303814 \\
9\end{array}$ & \\
\hline 8 & & & & & & & \\
\hline 9 & & & & \multirow{2}{*}{\multicolumn{2}{|c|}{ Indigène }} & 836866 & \\
\hline 10 & & & & & & & \\
\hline 11 & & & & \multicolumn{2}{|c|}{ Communautaire } & 110139 & \\
\hline 12 & \multirow{4}{*}{ Secondaire } & \multirow{4}{*}{1 à 3} & & \multicolumn{2}{|c|}{ Générale } & 3070865 & \multirow{4}{*}{92.5} \\
\hline \multirow{2}{*}{13} & & & & \multicolumn{2}{|c|}{ Technique } & 1714075 & \\
\hline & & & & \multicolumn{2}{|c|}{ Télé-enseignement $\left({ }^{* *}\right)$} & 1232982 & \\
\hline 14 & & & & \multicolumn{2}{|c|}{ Pour les travailleurs } & 375545 & \\
\hline 15 & \multirow{3}{*}{$\begin{array}{l}\text { Enseigne- } \\
\text { ment moyen } \\
\text { supérieur }\end{array}$} & \multirow{3}{*}{1 a 3} & \multirow{3}{*}{$\begin{array}{l}\text { Enseignement } \\
\text { moyen } \\
\text { supérieur }\end{array}$} & \multicolumn{2}{|c|}{ Professionnel-technique } & 352511 & 5.5 \\
\hline \multirow{2}{*}{16} & & & & \multirow[b]{2}{*}{ Baccalauréat } & Général & 2273762 & \multirow{2}{*}{53.3} \\
\hline & & & & & $\begin{array}{l}\text { Techno- } \\
\text { logique }\end{array}$ & 1116670 & \\
\hline 18 & & & 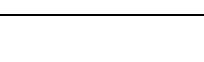 & \multirow{2}{*}{\multicolumn{2}{|c|}{$\begin{array}{c}\text { Technique supérieur } \\
\text { (2 à } 3 \text { ans) }\end{array}$}} & \multirow{2}{*}{80176} & \\
\hline 19 & & & & & & & \\
\hline 20 & & & & & $\begin{array}{l}\text { Enseigne- } \\
\text { ment normal }\end{array}$ & 136339 & \\
\hline 21 & $\begin{array}{c}\text { Enseigne- } \\
\text { ment }\end{array}$ & $\begin{array}{l}\text { du niveau } \\
\text { 12 à } 3 \text { ans }\end{array}$ & Enseignement & (4 à 5 ans) & $\begin{array}{l}\text { Universitaire } \\
\text { et technologi- } \\
\text { que }\end{array}$ & 2150146 & $\left.24.31^{* * *}\right)$ \\
\hline $\begin{array}{l}21- \\
24\end{array}$ & supérieur & $\begin{array}{l}\text { que supérieur; } \\
\text { licence, } 5 \text { ans }\end{array}$ & & & $\begin{array}{l}\text { Spécialité (un } \\
\text { an) }\end{array}$ & 34898 & \\
\hline 24 & & en moyenne) & & 3e cycle & $\begin{array}{c}\text { Maîtrise } \\
\text { (2 ans) }\end{array}$ & 111970 & \\
\hline $\begin{array}{l}\text { ef } \\
\text { plus }\end{array}$ & & & & & $\begin{array}{c}\text { Doctorat } \\
\text { (3 ans) }\end{array}$ & 15135 & \\
\hline
\end{tabular}

Source : élaboration de l'auteur sur la base de : INEE (2008). Panorama Éducatif du Mexique. Indicateurs du Système éducatif national. http://www.inee.edu.mx/index.php?option=com_content\&view=article\&id=3742\&Itemid=1070

* : y compris la population non comprise dans le groupe d'âge. **: Centres scolaires dirigés par deux enseignants qui coordonnent l'enseignement des trois niveaux et dont le contenu est réalisé avec l'appui de programmes nationaux de télévision et de livres et de matériels d'enseignement. *** SEP. Indicateurs et pronostics.

http://www.sep.gob.mx/wb2/sep1/sep1_Estadisticas 
orientée vers la formation pour le travail de la population jeune du pays, grâce aux institutions scolaires ; cet effort s'est traduit par la construction progressive d'un sous-système d'enseignement technologique, au cours des dernières quarante années, et par la forte orientation professionnelle de la formation universitaire (de Ibarrola, 2000).

La réforme éducative, initiée par le gouvernement fédéral en 1970, a été particulièrement importante à cet égard, dans la mesure où prennent naissance une grande partie des institutions à caractère fédéral qui, jusqu'à aujourd'hui, ont favorisé la formation scolaire pour le travail : le secondaire technique (7 à 9 ans : cinquième, quatrième et troisième modalités techniques); les baccalauréats bivalents (technologique et général) ; les Centres d'études technologiques (industriels et agricoles) et le Collège national d'éducation professionnelle technique (Conalep), tous couvrant la seconde, première et terminale). Leur objectif était de former «les professionnels techniciens de niveau intermédiaire » - travailleurs ou employés - qui occupaient des statuts équivalents (de niveau intermédiaire) sur les marchés du travail du pays dans tous les domaines économiques: l'agriculture, l'industrie, les services. Le système scolaire créa même un secteur économique supplémentaire : celui des sciences et technologies de la mer.

Le niveau intermédiaire de formation (lycée professionnel) pour le travail est né d'un constat établi à l'échelle nationale : celui de l'absence de ce type de statut au sein d'une pyramide professionnelle composée d'une large base de travailleurs possédant à peine le niveau primaire et d'un étroit sommet de travailleurs ayant une formation supérieure, sans soutien dans la partie intermédiaire de la pyramide (de Ibarolla, 1994a).

De nouveau à l'initiative du gouvernement fédéral, et à la suite de la création de l'Institut Polytechnique National en 1938, ont été créés en 1948, dans tout le pays, les instituts technologiques de niveau supérieur. Ils sont destinés à la formation professionnelle (niveau supérieur (bac +4 ) et récemment $3^{\text {e }}$ cycle) dans l'ingénierie, les administrations et les filières dédiées au secteur primaire de l'économie : agriculture et études vétérinaires puis technologies de la mer.
Depuis cette date, le sous-système d'éducation technologique se caractérise par une large diffusion géographique des opportunités de scolarité secondaire, intermédiaire et supérieure aux villes de taille moyenne du pays et aux zones rurales, scolarité conjuguée à une formation professionnelle spécialisée. L'argument recurrent étant de mettre la technique au service des classes populaires et du développement économique du pays. Cette décennie voit aussi la création d'universités publiques étatiques dans tous les États de la République.

Une seconde vague de réformes fut initiée dans les années 90, leur objectif explicite étant de « moderniser " l'Éducation nationale dans le contexte de l'entrée du pays dans la «globalisation » et de la signature notamment du Traité de libre commerce avec l'Amérique du Nord. Cela favorisa la création de nouvelles institutions scolaires de formation pour le travail, de niveau supérieur, gérées conjointement par le gouvernement fédéral et les gouvernements étatiques, au nom de la décentralisation initiée en 1993 : les Universités Technologiques (1991) (comme leurs homologues françaises) et les Universités Polytechniques, créées une décennie après (2002) destinées à permettre la poursuite des études vers la licence. Les voies offertes par ces nouvelles institutions se voulaient une réponse aux «nécessités économiques de la globalisation », avec un renforcement du contrôle des nouvelles technologies: informatique, bureautique, processus de production, maintenance industrielle, technologies de l'information et de la communication, électronique et automatisation, ou bien gestion des ressources naturelles, admininistration d'entreprises touristiques ou commerce extérieur et douanes.

Les universités et les instituts d'enseignement supérieur du pays se sont essentiellement consacrés à la formation aux professions. Les filières à visée professionnelle - les administrations diverses, la comptabilité, le droit, l'odontologie, la médecine, l'ingénierie de toute sorte - représentent la majorité des effectifs (plus de $90 \%$ ). De leur côté, les filières formant aux disciplines académiques comme les mathématiques, la physique, la biologie, la philosophie, les lettres, représentent moins de $10 \%$ des effectifs. La réforme des années 90 a fixé une série d'exigences en matière d'évaluation et d'organisation pour chaque institution d'enseignement supérieur 
afin d'améliorer la qualité de l'enseignement et d'apporter une réponse plus pertinente aux besoins professionnels liés à la globalisation (Kent, 1997). À tous les niveaux, il s'agissait de répondre aux nouveaux défis de la formation scolaire pour le travail grâce à une réforme ambitieuse qui proposait une " éducation basée sur les compétences » (éducation orientée par le « savoir faire » professionnel ou par les compétences professionnelles), conformément aux accords internationaux.

Les analyses précédentes évoquent les thèses selon lesquelles le système scolaire a été le grand planificateur des ressources humaines du pays ${ }^{3}$ et c'est ainsi qu'ont été proposées et systématisées les conditions de formation (contenus et durée) d'une série de statuts professionnels comme ceux de technicien intermédiaire, de professionnel technique de niveau intermédiaire, de technicien supérieur universitaire, de technicien spécialisé et ceux de toutes les professions dont l'exercice légal requiert une formation universitaire.

\section{Le lien avec le secteur professionnel}

Les décisions concernant les modalités et niveaux scolaires de formation pour le travail ne sont pas de l'exclusivité du gouvernement fédéral.

Au cours des années, en particulier en vertu des deux grandes réformes signalées, se sont instaurés différents mécanismes de "relation" avec le secteur productif, en particulier avec les organismes entreprenariaux nationaux et étatiques, avec l'espoir toujours renouvelé de fournir aux jeunes une formation pertinente et efficace, capable de répondre aux besoins des marchés du travail et du développement du pays. Parmi ces mécanismes : les Conseils étatiques et le Conseil National de Planification de l'Enseignement intermédiaire et supérieur, qui décident de la création des institutions mais aussi des carrières à offrir à partir d'études de prévisions régionales, des demandes du marché du travail et d'études

${ }^{3}$ Cette thèse constitue une partie essentielle du rapport « Expansion éducative et marché du travail », étude comparative réalisée en Allemagne, Espagne, France, Italie et Royaume-Uni (Béduwé et Planas, 2002). Postérieurement, entre 2002 et 2006, le Dr Planas a coordonné une étude similaire pour le Mexique (Planas, Mercado, Flores, Morales et de Ibarrola, 2004-2007). d'opportunité4 (Vargas, 2006 ; Mota Quintero, à paraître, Vega Tato, à paraître) ; la participation des entrepreneurs aux comités de direction des établissements ou aux comités de liaison de chaque école ; l'instrumentation de stages d'apprentisage obligatoires et des pratiques professionnelles dans les entreprises ; les études de suivi de diplômés ; quelques stratégies de gestion mises en place au sein des institutions scolaires, comme la coopérative scolaire de production, la formation des entrepreneurs, le développement de projets productifs, la participation à des concours nationaux de créativité.

La participation du secteur professionnel n'a pas engendré alors, de la part des entreprises ou des secteurs économiques, une planification systématique des ressources humaines dont le pays avait besoin ; cette planification s'est cependant expimée de façon indirecte puisque les fonctionnaires du système éducatif ont traduit et systématisé les besoins qu'ils percevaient dans le secteur professionnel ou qui éventuellement leur étaient signalés par certains de leurs représentants 5 .

Par ailleurs, dans le contexte d'un système scolaire essentiellement public au niveau élémentaire (92\%), on ne peut occulter l'augmentation du nombre des institutions privées d'enseignement supérieur qui, de $15 \%$ des effectifs dans les années 80 , passèrent à $32,5 \%$ en 2006. Les presque 1500 institutions privées d'enseignement supérieur qui existent dans le pays façonnent un univers très différent, faiblement régulé, et répondent essentiellement aux intérêts et à la demande croissante des jeunes de niveaux socioéconomiques très divers dans toutes les villes du pays, jeunes qui ne trouvent pas de place dans le système d'enseignement supérieur (Vega Tato, à paraître).

Chaque sous-système mais aussi chaque établissement scolaire devient alors - selon son histoire, ses objectifs, le degré de consolidation de ses ressources humaines et matérielles et les procédés et pratiques éducatives qu'il a réussi à consolider - un médiateur

\footnotetext{
${ }^{4}$ Nous ne trouvons aucune mention relative à la participation des syndicats dans ces conseils.

${ }^{5}$ Le sous-secrétaire à l'Enseignement supérieur a annoncé l'ouverture de nouvelles filières pour les Universités Technologiques qui ont été créées après 160 réunions de consultation avec 1600 entreprises représentatives.
} 
important entre la politique du gouvernement, les intérêts exprimés par le secteur entreprenarial et la demande des jeunes.

Il s'agit alors d'une planification «atomisée » des ressources humaines requises par le pays, parmi d'autres objectifs poursuivis par les institutions scolaires, et initiée par des intérêts très différents. Mais cette planification est aussi « centralisée » dans la mesure où, à l'exception des universités autonomes, les décisions finales sur les filières ou programmes d'études et leur accréditation et certification reviennent aux structures éducatives du gouvernement fédéral et, récemment, des gouvernements étatiques.

\section{L'INFLUENCE DES MARCHÉS DU TRAVAIL : HÉTÉROGÉNÉITÉ ET INÉGALTÉS}

Un des problèmes les plus ardus pour la planification et l'organisation de la formation scolaire des ressources humaines est la méconnaissance de la nature des marchés du travail du pays et de leur lien à la force de travail scolarisée du pays.

Parmi les chercheurs de cette thématique, l'adhésion à la thèse de la coexistence de marchés hétérogènes du travail au Mexique est grandissante, alimentée par la constante et visible tension entre la possibilité d'établir des espaces de travail régulés, conformément à une législation professionnelle ambitieuse ${ }^{6}$, et la nécessité - et la possibilité - de générer des types d'activités très divers qui procurent des revenus à ceux qui ne peuvent s'insérer dans ces espaces régulés, indépendamment du secteur économique concerné. On peut qualifier les premiers d'espaces « formels » de travail et les seconds d' « informels »7.

\footnotetext{
${ }^{6}$ La constitution politique et la législation professionnelle comportent un grand nombre de conditions légales du travail très favorables aux " ouvriers, paysans et classes moyennes du pays »; cependant, à aucune période, il n'a été possible d'intégrer l'ensemble de la population dans ce cadre légal.

${ }^{7}$ Le concept de secteur informel, largement développé en Amérique latine, a été introduit dans les études sur ce thème à partir du rapport de l'OIT (Organisation internationale du Travail) sur le Kenya en 1972.
}

La présence et la coexistence d'espaces aussi inégaux constituent une des expressions les plus nettes de l'inégalité sociale et économique qui règne au sein de la population du pays et même si ces espaces assurent une activité professionnelle rémunérée à la population, ils contribuent à expliquer l'inégalité des revenus et la croissance faible, irrégulière et même négative du PIB (produit intérieur brut) au cours des années étudiées; ils déterminent les relations erratiques avec la scolarité.

Dans le secteur formel de l'économie, les droits à des contrats formels et à des conditions élémentaires légales sont respectés : revenus minimaux garantis, salaires régulés par des grilles strictes, sécurité sociale, étrennes ou primes de vacances et la fin du contrat comme ultime option et dans des conditions très favorables aux travailleurs. Le secteur formel se caractérise par une division technique et hiérarchique du travail, relativement complexe, qui favorise ceux qui exercent des professions ou possèdent des statuts correspondant aux exigences en matière de scolarité (de Ibarrola, 2006). On trouve ici les entreprises qui accordent une attention particulière aux changements technologiques de «frontière ", aux nouvelles demandes sociétales et aux réglementations qui comportent, à l'ouverture de l'économie, des innovations relatives aux produits et aux procédés et au rôle essentiel de la connaissance dans la production (Planas, 2008).

Le «secteur informel de l'économie » concerne la multitude d'emplois et d'activités qui ne font pas partie de ces diverses dimensions de la légalité professionnelle, qui visent à obtenir des revenus individuels et familiaux permettant la survie de nombreux groupes de population du pays. Il comporte de nombreux "cuentapropistas " (travailleurs à leur compte) qui se consacrent à toute sorte de commerces et de services précaires, qu'on aperçoit d'un simple coup d'œil à tous les coins de rue et artères importantes des villes. Il comprend les petites unités domestiques de production en milieu rural et la plupart des petits ateliers artisanaux ou des micro-entreprises industrielles ou de commerce de moins de cinq travailleurs, qui concernent le vaste et varié ensemble d'activités indispensables qui ne correspondent pas aux statuts professionnels tels qu'ils sont définis par le système scolaire. Les activités productives et les 
horaires sont ici indifférenciés et s'imbriquent dans les divers aspects de la production et reproduction des familles et l'activité domestique. Le secteur informel se caractérise par l'utilisation d'une technologie obsolète ou très rudimentaire, l'absence de capital, la faible formation de la force de travail, une scolarité très restreinte, des conditions de travail très précaires conjuguées à de modestes revenus (Gallart, 2004).

Bien qu'insuffisante, cette division conceptuelle permet de dépasser la notion selon laquelle, dans le pays, un seul type de logique est à l'œuvre en matière de création d'emplois. Selon une base de données nationales pour le Mexique, élaborée par l'Institut International de Planification de l'Éducation de l'UNESCO (IIPE) qui, conformément à cette distinction clé des marchés du travail, met en relation la scolarité avec les revenus, on peut identifier cinq secteurs professionnels, trois secteurs formels et deux secteurs informels, et analyser leur relation avec la scolarité de la population (de Ibarrola, 2006) ${ }^{8}$.

Au sein du secteur formel de l'économie, le marché du travail public se démarque : y dominent les opportunités professionnelles qui favorisent son propre système scolaire dans tous ses niveaux et ses modalités, et une structure très restreinte pour la recherche scientifique ; la sécurité sociale en matière de santé et d'autres prestations professionnelles et une multitude de programmes gouvernementaux d'aide, par exemple à la production agricole ou au logement et à la consommation populaires. Selon les données disponibles, le secteur public emploie actuellement $6 \%$ de la population active de 25 à 60 ans.

Sont également qualifiées de formelles les entreprises industrielles et de services de plus de cinq salariés. Selon ces chiffres, ces deux secteurs représentent $54 \%$ de la population active.

Selon les données évoquées, le secteur informel de l'économie correspond à $34,6 \%$ de la population active, $8,8 \%$ concernant les travailleurs à leur

\footnotetext{
${ }^{8}$ Les données examinées ont été produites par l'IPPE (Institut International de Planification de l'Éducation) de Buenos Aires, systématisées pour réaliser un comparatif entre les pays d'Amérique latine. Elles se sont appuyées sur une sélection d'informations de l'Enquête nationale sur les ménages. Elles n'intègrent pas la polulation active de moins de 24 ans ni celle du secteur primaire.
}

compte et 25,8 \% les salariés et patrons d'entreprises de moins de cinq travailleurs.

\section{Les liens entre la scolarité et le travail}

Dans cette vision d'ensemble des marchés du travail du pays, marchés différenciés et inégaux, il est possible de faire ressortir quelques résultats globaux sur lesquels se fondent, généralement, les attentes par rapport au rôle de la scolarité.

Il ressort ainsi que les revenus de la population active s'accroissent de manière substantielle avec l'augmentation de la scolarité. Selon les données fournies par l'IIPE, tandis que les travailleurs qui ont suivi une scolarité de 5 ans au maximum (scolarité primaire) gagnent à peine la moitié du revenu moyen de la population active, ceux qui ont suivi un enseignement supérieur (complet ou incomplet avec 13 ans de scolarité ou plus) gagnent deux fois ce salaire moyen (de Ibarrola, 2006). ${ }^{9}$

Indépendamment des conditions de travail inégales qui s'observent au sein des différents secteurs, un meilleur niveau de scolarité assure de meilleurs revenus dans chaque secteur. L'écart des revenus entre ceux dont la scolarité est élevée et ceux qui ont été moins scolarisés est évidente dans les cinq secteurs professionnels identifiés : avec un minimum de 1,25 fois parmi les travailleurs à leur compte et un maximum de 3,7 fois parmi les travailleurs des grandes entreprises du secteur secondaire; même entre salariés et patrons de micro-entreprises de moins de cinq travailleurs, la différence est de 3,3 fois. Les données précédentes révèlent aussi l'inégalité de revenus entre ceux qui, possédant le niveau le plus élevé de scolarité, travaillent dans le secteur informel ou formel.

Dans les rares études qui mettent en relation ces aspects des marchés du travail avec la scolarité des travailleurs, l’hétérogénéité des politiques de recrutement ressort. Les secteurs formels du marché du travail accorderaient de la valeur à la scolarité en termes de revenu, même si on observe des différences entre les entreprises qui font un usage intensif

${ }^{9}$ Ces corrélations n’invalident pas différentes variables : sociales, économiques, culturelles, concernant ceux qui font partie des groupes possédant ce niveau de scolarité, qui se modifient avec l'augmentation des opportunités scolaires. 
de la main-d'œuvre et celles à forte intensité capitalistique, différences liées au sexe, à l'âge, à l'expérience professionnelle antérieure, au secteur de l'économie, à la zone géographique du pays ou aux entreprises elles-mêmes (de Ibarrola, 2004 ; Planas, 2008 ; Hualde y Serrano, 2005). C'est dans ces espaces formels que l'on trouvera le "sous-emploi instruit ", et de grandes différences de revenus et de statuts entre les travailleurs qui ont suivi une même scolarité ; on y découvrira aussi la difficulté rencontrée par les jeunes pour accéder à des statuts et des secteurs qui leur étaient pourtant promis par la poursuite d'études (Suarez Zozaya, 2005). Certes, sur les marchés informels, il n’y a pas d'exigences en matière de scolarité ; cependant, en raison de l'augmentation de la scolarité de la population active, la scolarité de cette population de travailleurs informels a aussi augmenté et, parmi eux, ceux qui possèdent une meilleure scolarité sont aussi mieux rémunérés (de Ibarrola, 2006 ; Planas, 2008).

Considérer les marchés du travail du pays comme hétérogènes et inégaux permet d'expliquer pourquoi les mécanismes d'articulation prévus par le système scolaire n'ont pas favorisé une organisation fonctionnelle. Même si une meilleure scolarité est reconnue du point de vue financier, elle n'est pas forcément liée à la nature et au contenu des activités professionnelles ; à cet égard, 51 \% des diplômés du Conalep (2006) (enseignement intermédiaire) indiquent que leur travail correspond peu ou pas du tout à la filière qu'ils ont suivie et, en moyenne, 32 \% de la population active ayant suivi une scolarité supérieure exercent des activités qui ne correspondent pas à leur formation (Observatoire professionnel, 2008).

Cette hétérogéneité fausse toute étude sur l'augmentation des besoins en matière de force de travail et, plus encore, toute étude sur la différence d'augmentation de ces besoins selon les niveaux de scolarité liés aux hiérarchies dans le travail. Elle signifie que les statuts professionnels dont les limites et les contenus sont déterminés par le système scolaire n'existent pas ou ne sont pas recherchés de façon générale sur les marchés du travail. D’un autre côté, les relations entre écoles et entreprises restent assujetties au type d'entreprises locales avec lesquelles s'établissent les relations, nombre d'entre elles étant soumises à des degrés d'informalité et de précarité différents. Malgré l'accroissement de la scolarité formelle de la population, le secteur professionnel informel s'est développé au cours de la dernière décennie.

\section{LES INTÉRÊTS DES JEUNES ET DE LEURS FAMILLES}

Globalement, les données précédentes semblent correspondre aux représentations des étudiants et de leurs familles par rapport à la scolarité qui leur convient. Le fait est que les jeunes privilégient l'accès à l'enseignement supérieur (même s'ils n'y parviennent pas), optent pour certaines modalités, domaines de connaissance et professions spécifiques. Ces choix sont dictés par les expériences familiales, les contacts professionnels immédiats et la publicité dans les médias. Ils révèlent aussi l'absence d'une politique efficace d'orientation professionnelle au collège et même au lycée.

\section{L'intérêt manifeste pour l'accès à l'enseignement supérieur}

Les statistiques indiquent que $90 \%$ de ceux qui s'inscrivent au niveau intermédiaire supérieur optent pour les divers types de bac qui conduisent à l'enseignement supérieur.

Très récemment, $80 \%$ de ceux qui ont atteint ce niveau ont poursuivi vers le supérieur, même si nombre d'entre eux ont abandonné au cours des premiers trimestres.

En revanche, le Collège National d’Éducation Professionnelle Technique (Conalep), qui a essayé de canaliser directement les jeunes vers le marché du travail en proposant douze ans de scolarité, n’a pas réussi à rassembler plus de $10 \%$ des effectifs du niveau, et au cours des dernières années, ce pourcentage a diminué en valeur absolue; dès 1993, le Collège National a offert la possibilité d'obtenir le baccalauréat en plus du BEP.

Le désintérêt des jeunes pour ce type d'études peut s'expliquer par le destin professionnel informel et précaire qui les attend. Selon le rapport national 20022005 du Conalep, 65,3 \% des diplômés travaillaient 
au moment de l'enquête ${ }^{10}$ (même si certains, 24,1 \%, n'avaient pas trouvé de travail au cours d'une année), le reste continuant à étudier. Parmi ceux qui travaillent, seulement $74,4 \%$ perçoivent un salaire fixe, $67 \%$ reçoivent les prestations minimales, $88 \%$ gagnent entre une et trois fois le salaire minimum, $80 \%$ travaillent dans le secteur privé, $26 \%$ dans des entreprises de moins de cinq travailleurs et - comme on l'a évoqué - $51 \%$ indiquent que leur travail correspond peu ou pas du tout à la filière suivie (Conalep, 2006).

De leur côté, plus de quinze ans après leur création, les Universités Technologiques n’ont pas davantage convaincu les jeunes des avantages de cette formation et elles n'atteignent toujours pas l'effectif prévu de 150000 étudiants, mais à peine $41,6 \%$ de celui-ci (Rubio Oca, 2006). Dès 2002, ont été créées 23 Universités Polytechniques prévues pour 115000 étudiants qui, tout en offrant la même formation de technicien supérieur, permettent de commencer des études de licence, augmentant ainsi les années d'études. À ce jour, on dénombre 18000 étudiants, à peine $15,7 \%$ de ce qui était prévu, même si cela peut s'expliquer par le rythme naturel de croissance des institutions scolaires et le temps dont elles ont besoin pour démontrer leur efficacité.

Le taux d'abandon des jeunes avant la fin de la trajectoire programmée par les institutions scolaires, tant au niveau intermédiaire qu'au niveau supérieur, est très élevé : entre 45 et $55 \%$ (SEP, 2008). Cette statistique est régulièrement utilisée pour justifier la création de modalités d'études courtes, bien que cela ne semble pas constituer un argument suffisant pour les jeunes, qui semblent préférer une durée d'études plus courte qu'ils parviennent à suivre au sein d'institutions plus traditionnelles.

\footnotetext{
${ }^{10}$ Enquête de 2006 sur un échantillon aléatoire de diplômés des générations 2001-2004 et 2002-2005 stratifié par filières et régions. L'échantillon était de 1833 diplômés avec un intervalle de confiance de $95 \%$ et d'erreur maximale de $2.29 \%$ pour les résultats nationaux. Cette enquête est sous la responsabilité du système de suivi des diplômés du CONALEP.
}

\section{La préférence des étudiants pour certains domaines de connaissance et filières}

Par ailleurs, au niveau supérieur, deux types de chiffres conduisent à considérer différemment les interactions et tensions qui structurent la formation scolaire: la préférence des étudiants pour les domaines des sciences sociales et administratives et l'ingénierie, et en particulier pour sept filières. Au niveau intermédiaire, on note aussi que les jeunes optent pour les mêmes domaines, mais nous ne disposons pas d'information suffisante pour approfondir la question.

Le tableau 1 présente la répartition en pourcentage du total des étudiants de l'enseignement supérieur selon les domaines de connaissance ${ }^{11}$, à trois dates différentes. Pour 2006, on additionne l'information, ce qui permet de comparer cette répartition selon trois critères : dans les institutions privées, au sein des nouvelles universités technologiques, et dans le $3^{\text {e }}$ cycle national (maîtrise et doctorat).

Un autre résultat notable est la préférence des étudiants pour sept filières qui représentent $50 \%$ des inscriptions. Ces filières fournissent en effet plus de $50 \%$ des emplois de professionnels dans le pays, bien que l'on observe des décalages qui méritent un examen plus approfondi, puisque la première donnée concerne le stock de professionnels ${ }^{12}$ sur les marchés du travail et le second le flux d'étudiants. Signalons qu'une forte proportion de chacun de ces groupes professionnels exerce des activités étrangères à leur formation ${ }^{13}$, situation qui ne modifie pas la moyenne des revenus les plus élevés, corollaire du niveau élevé de scolarité (ANUIES, 2003, pp. 181-197).

\footnotetext{
${ }^{11}$ D'après la classification de l'Association Nationale d'Enseignement Supérieur.

${ }^{12} \mathrm{Au}$ Mexique, on appelle "profesionistas » les travailleurs qui ont un niveau de scolarité supérieur. Les emplois professionnels requièrent une scolarité de niveau supérieur.

${ }^{13}$ Il s'agit de la différence entre ceux qui sont classés dans leur profession, et ceux qui exercent au sein de groupes différents ou dans des activités où la hiérarchie est moindre. Cette situation affecte en réalité toutes les professions du pays.
} 
Tableau 1

Répartition en pourcentage du total des étudiants de l'enseignement supérieur selon les disciplines, 1970-2006

\begin{tabular}{|c|c|c|c|c|c|c|}
\hline $\begin{array}{l}\text { Domaines de } \\
\text { connaissance }\end{array}$ & $\begin{array}{c}1970 \\
\%\end{array}$ & $\begin{array}{c}1984 \\
\%\end{array}$ & $\begin{array}{l}2006 \\
\%\end{array}$ & $\begin{array}{l}\text { Institutions } \\
\text { privées } 2006\end{array}$ & $\begin{array}{c}\text { Universités } \\
\text { technologiques } \\
2006 \\
\%\end{array}$ & $\begin{array}{c}\text { 3e cycle } \\
2006 \text { (total) } \\
\%\end{array}$ \\
\hline Sciences agricoles & 3,7 & 9,5 & 2,1 & 0,3 & 1,1 & 1,7 \\
\hline Sciences de la santé & 19,6 & 14,5 & 8,9 & 5,6 & 4,2 & 14,7 \\
\hline $\begin{array}{c}\text { Sciences naturelles } \\
\text { et exactes }\end{array}$ & 0.9 & 3,0 & 1,8 & 0,3 & 0 & 5,1 \\
\hline $\begin{array}{l}\text { Sciences sociales } \\
\text { et administratives }\end{array}$ & 43,5 & 42,8 & 44,2 & 63,6 & 37,0 & 45,5 \\
\hline $\begin{array}{l}\text { Enseignement } \\
\text { et sciences humaines }\end{array}$ & 4,1 & 2,9 & $\left.11,61^{*}\right)$ & 6,6 & 1,8 & 21,5 \\
\hline \multirow[t]{2}{*}{$\begin{array}{l}\text { Ingénieries } \\
\text { et technologies }\end{array}$} & 28,2 & 27,3 & 31,4 & 23,9 & 55,9 & 11,5 \\
\hline & 100,0 & 100,0 & 100,0 & 100,0 & 100,0 & 100,0 \\
\hline Total & 251054 & 939513 & 2150146 & 712648 & 80176 & 162003 \\
\hline
\end{tabular}

Source : pour 1970, SEP/ANUIES. Plan national de l'enseignement. Pour 1984 : ANUIES. Annuaire statistique de l'Association nationale des universités correspondantes ; pour 2006 : ANUIES. Statistiques de l'Enseignement supérieur. http://www.anuies.mx/servicios/e_educacion/index2.php

$\left({ }^{*}\right)$ : Y compris les chiffres correspondant aux écoles Normales, qui passèrent au niveau supérieur en 1984.

Tableau 2

Répartition en \% de $50 \%$ des étudiants par rapport au pourcentage des emplois professionnels du pays, et \% des employés professionnels dont le contenu du travail correspond à la filière d'études suivie

\begin{tabular}{|l|c|c|c|}
\hline \multicolumn{1}{|c|}{ Filières } & $\begin{array}{c}\text { \% par rapport au total } \\
\text { de professionnels }\end{array}$ & $\begin{array}{c}\text { \% des effectifs de } \\
\text { I'enseignement supérieur }\end{array}$ & $\begin{array}{c}\text { \% de professionnels } \\
\text { de la filière employé } \\
\text { dans leur spécialité }\end{array}$ \\
\hline Comptabilité & 12,1 & 7,1 & 63,5 \\
\hline $\begin{array}{l}\text { Enseignement de base, } \\
\text { secondaire et normal }\end{array}$ & 10,9 & 5,9 & 75,6 \\
\hline Administration & 8,2 & 10,9 & 43,2 \\
\hline Droit & 7,9 & 10,7 & 66,3 \\
\hline $\begin{array}{l}\text { Ingénierie mécanique } \\
\text { et industrielle }\end{array}$ & 5,4 & 5,3 & 40,1 \\
\hline Médecine & 3,4 & 4,1 & 88,1 \\
\hline $\begin{array}{l}\text { Ingénierie, systèmes } \\
\text { informatiques }\end{array}$ & 3,3 & 6,4 & 45,5 \\
\hline Participation de ces filières & 51,2 & 50,4 & \\
\hline
\end{tabular}

Sources : http://www.observatoriolaboral.gob.mx y ANUIES (2008). Annuaire statistique 2005-2007. 


\section{TENSIONS ET CONTRADICTIONS}

Les données des tableaux précédentes (tableaux 1 et 2) reflètent la structure actuelle de la formation scolaire pour le travail. Ils sont le résultat d'une longue histoire d'interactions, de tensions et de contradictions entre les acteurs évoqués, dont l'explication exige l'étude de mécanismes et de variables beaucoup plus complexes que ceux que nous proposons ici comme première approche du problème.

Le gouvernement fédéral a soutenu l'accroissement des opportunités scolaires, arguant toujours de leur importance pour le développement économique du pays, la nécessité d'anticiper le type et le niveau de formation de la force de travail qui seraient demandés par le marché du travail du pays, la réponse aux besoins sociaux de la population ou l'impulsion donnée au potentiel économique de nombreuses ressources naturelles.

Mais l'intervention du secteur professionnel et les demandes des jeunes permettent de conclure que, audelà de l'identification d'un «besoin social à combler» ou des «demandes» d'un marché du travail constitué d'une partie à peine des secteurs professionnels du pays, ce sont les structures effectives de travail et d'emploi - ou l'information qui circule à ce sujet - qui définissent la structure résultant des opportunités scolaires, issue de l'interaction entre ce que les institutions scolaires offrent en pratique et la demande des jeunes.

Un examen approfondi des données précédentes permet de valider cette affirmation.

\section{Sciences agricoles}

La création des bacs technologiques agricoles et des instituts technologiques agricoles dans les zones essentiellement rurales s'inscrit dans cette volonté du gouvernement fédéral. Cependant, même la forte proportion de la population active qui travaille encore dans le secteur primaire (13,5\%) n'a pu empêcher la diminution notoire des effectifs en sciences agricoles, qui représentaient 9,4\% des effectifs dans les années 80 et ont chuté à 2,3\% actuellement. Derrière cette variation, on trouve l'ouverture et l'arrêt d'importants programmes gouvernementaux d'aide à l'agriculture mexicaine, l'affaiblissement de l'État et par conséquent la fermeture des marchés du travail que seul le gouvernement offrait à ce type de professions (de Ibarrola, 1994a ; Gastelum, 2008). Les nouvelles institutions publiques créées dans les années 90 n’ont pas favorisé ce domaine, pas plus que ne l'a fait le $3^{\text {e cycle. }}$ Le secteur privé de l'enseignement supérieur ne proposait quasiment pas ce type de filière.

\section{Sciences de la santé}

Les filières des sciences de la santé ont largement réduit leur participation à l'effectif scolaire malgré les besoins d'une population de plus de 100 millions d'habitants. En raison d'un marché du travail réduit, géré presque dans sa totalité, et avec de très faibles ressources budgétaires, par le secteur public - assistance publique gratuite ou sécurité sociale, et avec un secteur privé très restreint et coûteux - ont été introduits deux procédés stricts de sélection qui ont conduit à décourager et à rejeter la demande, l'un lors de l'admission à la filière et l'autre lors de l'accès aux stages dans les hôpitaux. La forte proportion de l'effectif du 3e cycle dans ce domaine s'explique par l'exigence croissante d'une meilleure spécialisation dans ces professions. Ce groupe professionnel est le plus attaché à l'exercice de la profession pour laquelle il a étudié, comme il est à la fois le plus contrôlé par le gouvernement et par sa propre corporation.

\section{Sciences naturelles et exactes}

Tous les discours sur l'économie de la connaissance et le rôle de la science comme amorce du changement technologique, qui caractérisent le développement global, n’ont semble-t-il pas suffit à accroître l'effectif dans ce domaine. Les mathématiques représentent un total national de 9667 étudiants et la physique 3 382, et un effectif quasi nul dans les institutions privées. Les nouvelles universités ne proposent tout simplement pas ces filières. Une explication réside dans la réduction des opportunités de travail pour leurs diplômés, dont l'employabilité se concentre essentiellement dans le secteur éducatif, ou dans les faibles opportunités en matière de recherche scientifique offertes par le secteur académique public, 
ce qui explique la forte proportion du $3^{\text {e cycle, }}$ puisque pour l'emploi de chercheur le doctorat est exigé14.

\section{Sciences sociales et administratives}

Presque la moitié des étudiants mexicains de niveau supérieur se concentre dans les filières du domaine des sciences sociales et administratives, tout au long

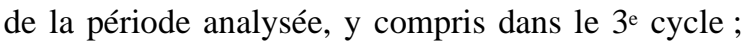
cela semble lié à un ensemble de facteurs explicatifs : la grande tradition historique des trois professions explique cette concentration, en particulier celle du droit ; le fait que leurs diplômés trouvent effectivement du travail et obtiennent de meilleurs revenus, même si dans une large mesure l'activité exercée n'est pas en lien avec la formation suivie ; le coût relativement réduit engendré par l'offre de ce type de formation; le prestige de ces professions intellectuelles très liées au leaderschip politique du pays ; l'absence d'une orientation et d'une information professionnelles efficaces. Il est important de signaler que, dans ce domaine, on trouve de nombreuses filières qui correspondent à des nouvelles spécialisations.

\section{Enseignement et sciences humaines}

L'augmentation du nombre des filières d'enseignement s'explique par l'accroissement du niveau de scolarité exigé pour être enseignant. En effet, pour les enseignants de l'enseignement élémentaire, à partir de 1984, on exige la licence ; par ailleurs, les enseignants du niveau intermédiaire et supérieur ont été incités à obtenir licences, maîtrises et doctorats par l'octroi de primes au mérite. Cependant, le marché du travail offert par le système scolaire, en particulier au niveau élémentaire, est l'un des plus régulé par le budget public et comme on l'a vu, il ne permet pas de répondre aux besoins d'éducation de la population.

\footnotetext{
${ }^{14}$ Le pays consacre moins de $0,4 \%$ du PIB à la recherche scientifique. La participation du secteur des entreprises est minime, illustration de la dépendance scientifique et technologique.
}

\section{Ingénierie et technologies}

L’impulsion constante donnée par le gouvernement à la formation de techniciens intermédiaires et d'ingénieurs dans diverses branches de l'ingénierie, en particulier l'informatique et l'électronique, depuis les années 70 , a été fructueuse. Actuellement, cette branche représente plus de $30 \%$ des effectifs et dans certaines institutions publiques, elle atteint plus de 50 \%. De même, elle a permis d'anticiper le besoin de ressources humaines formées, par exemple à la frontière avec les États-Unis, où le renforcement de l'industrie délocalisée en électronique et informatique a largement bénéficié de la formation de travailleurs dans ces domaines (Hualde et Serrano, 2005). Cela explique pourquoi, dans ce domaine, le taux de diplômés dont l'activité professionnelle correspond à la spécialité de formation est des plus bas.

La création des universités technologiques et polytechniques centrées sur l'offre de ce type de filières traduit la double vision du gouvernement fédéral : étendre les opportunités de scolarité aux régions très isolées du pays et faire de la scolarité le moteur du développement économique. La situation actuelle est un exemple des divergences possibles à court et moyen terme entre la planification scolaire, l'intérêt des étudiants et les possibilités d'exercer des activités correspondant aux formations suivies.



Le système scolaire mexicain, géré par le gouvernement fédéral, a constitué la voix privilégiée pour définir et organiser la qualification des ressources humaines du pays. Mais dans les faits, la structure actuelle de la formation scolaire pour le travail est le fruit des tensions et contradictions historiques entre les propositions du gouvernement fédéral en matière éducative, les interventions indirectes et très limitées du secteur entreprenarial, le degré de stabilité des institutions scolaires elles-mêmes et les intérêts et motivations des élèves.

Ces interactions - très peu étudiées - expriment en particulier la nature des conditions de travail réelles qui prévalent dans le pays, en raison de la structure hétérogène et inégale de ses marchés du travail. Elles 
se sont traduites par des situations contradictoires qui conjuguent l'accroissement notable de la scolarité et l'insuffisante prise en compte de la population adulte de plus de 15 ans ; la création d'institutions scolaires et l'offre de filières qui n'emportent pas l'adhésion des jeunes ; la baisse des effectifs de filières destinées à répondre à d'énormes besoins sociaux, pour lesquels il n'existe pas de structures de travail suffisantes. De cela, il résulte non seulement une saturation de filières pour lesquelles il existe des opportunités de travail même si l'activité professionnelle s'exerce uniquement dans des contextes et fonctions éloignés de la formation, mais aussi une grande différence de revenus au profit de ceux qui possèdent de meilleurs niveaux de scolarité, en particulier dans l'enseignement supérieur. Malgré l'augmentation notable de la scolarité de la population active, le secteur informel de l'économie s'est développé, englobant une forte proportion de la population qui possède une scolarité supérieure, et l'accroissement du PIB n'a pas cessé de se réduire, PIB erratique et même négatif.

La volonté du gouvernement fédéral de créer de nouvelles institutions de formation scolaire pour le travail ne peut pas occulter les profondes inégalités professionnelles qui règnent dans le pays et les inégalités sociales, économiques, politiques et culturelles qu'elles reflètent.

L'analyse développée dans cet essai conforte la nécessité de recherches qui reconnaissent la complexité des mécanismes sur lesquels s’appuient les propositions gouvernementales, le rôle joué par les autres acteurs concernés et la nature des réalités auxquelles ils sont confrontés. Il s’agit d'évaluer les institutions créées, les obstacles qu'elles rencontrent, les solutions qu'elles trouvent et les effets à moyen et à long terme. Les données disponibles montrent que la priorité accordée par l'État mexicain à la scolarité de la population active et à la formation scolaire pour le travail comme mécanisme de développement du pays a été insuffisante et a été rattrapée, dans une large mesure, par des problèmes de développement économique, d'équité et de justice sociale.

Traduit de l'espagnol au français par Mireille Zangani (Céreq), avec l'aimable collaboration de l'auteur.

\section{Bibliographie}

Aguilar Villanueva L. (ed.) (1993), Problemas públicos y agenda de gobierno. México: Editorial Miguel Ángel Porrua.

Anuies (Asociación Nacional de Universidades e Institutos de Educación Superior), (1984, 1994). Anuario estadístico. México.

Anuies (2003), Mercado laboral de profesionistas en México. Diagnóstico 1990-2000. México, vol. 4.

Béduwé C. y Planas J. (2002), Expansión educativa y mercado de trabajo. Estudio comparativo realizado en cinco países europeos: Alemania, España,
Francia, Italia, Reino Unido, con referencia a los Estados Unidos. Informe final de un proyecto financiado por el Programa Marco de Investigaciones y Desarrollos de la Unión Europea: Ministerio de trabajos y asuntos sociales. Instituto Nacional de las Cualificaciones de Madrid.

Boudon R. (1980), Efectos Perversos y orden social. México. Premia.

Colegio Nacional de Educación Profesional Técnica, (2006). Seguimiento de Egresados. Informe Nacional Encuesta 2001-2004 y 2002-2005, SEP/Conalep/ Rvox/IPSolutions, México. 
De Ibarrola M. (1994 a), Escuela y trabajo en el sector agropecuario en México. México: CINVESTAV/ Instituto José María Luís Mora/ Porrúa/ FLACSO.

De Ibarrola M. (1994 b), Industria y escuela técnica. Dos experiencias mexicanas. UNESCO-OREALC/ Red Latinoamericana de educación y trabajo. Santiago, Buenos Aires, México.

De Ibarrola M. (dirección) (2004), Escuela, capacitación y aprendizaje. La formación para el trabajo en una ciudad en transición. Montevideo. Cinterfor/ OIT, 222 p. (Herramientas para la transformación, 27).

De Ibarrola M. (2006), Formación escolar para el trabajo. Montevideo. CINTERFOR, 2006. (Sobre Artes y Oficios, 5) 411 pp. ISBN: 92-9088-213-1 http://www.cinterfor.org.uy/public/spanish/region/ ampro/cinterfor/publ/arte/ibarro/index.htm

De Ibarrola M. (2006), El incremento de la escolaridad y sus efectos sobre el mercado de trabajo en México, 1992-2004. Informe del caso mexicano. Siteal/UNESCO-IIPE/OEI. Documento no publicado.

Gallart M. A. (2004), "Habilidades y competencias para el sector informal de la economía”. Formación en la economía informal. Boletín Cinterfor (155), pp. 33-75.

Gastelum J. (2008), La profesión agronómica ante la situación de la agricultura mexicana, en la perspectiva de la apertura comercial y el adelgazamiento del Estado. Tesis para obtener el grado de Doctor en Ciencias en la especialidad de Investigaciones Educativas. DIE-Cinvestav IPN. México.

Hualde A. y Serrano A. (2005), "La calidad del empleo de asalariados con educación superior en Tijuana y Monterrey”. Revista Mexicana de Investigación Educativa (México), vol. 10, n 25, pp. 345374.

Instituto Nacional para la Evaluación de la Educación (2005), Panorama educativo de México. 2005. Indicadores del sistema. INEE, México. pp. 54999.
Kent R. (compilador) (1997), Los temas críticos de la Educación superior en América Latina. FLACSO /Fondo de Cultura Económica. Santiago/México. 2 vol.

Mercado A. y Planas J. (2005), “Evolución del nivel de estudios de la oferta de trabajo en México. Una comparación con la Unión Europea”. Revista Mexicana de Investigación Educativa, (México) vol. 10, n 25, pp. 315- 343.

Mota Quintero A. (2008), Las Universidades tecnológicas de desarrollo. Un caso de formación laboral por competencias. Proyecto de tesis de doctorado en ciencias con especialidad en Investigación Educativa. DIE-CInvestav. Tesis en proceso.

Observatorio Laboral (2008): www.observatoriolaboral.gob.mx/ola/ola-panorama-_del_-desempleo.

Pieck E. (2005), "La secundaria técnica. Su contribución a la formación para el trabajo en sectores de pobreza”. En Revista Mexicana de Investigación Educativa (México) vol. 10, n² 25 pp. 481-507.

Planas J., Mercado A., Flores R, Morales I. y de Ibarrola M. (2007), Expansión educativa y mercado de trabajo en México. Una comparación con la Unión Europea. Documentos de trabajo elaborados en el marco de una investigación colectiva financiada por la Universidad de Guadalajara. No publicados.

Planas J. (2008), El comportamiento de los empleadores mexicanos frente al crecimiento de la educación. Revista de Educación Superior. México, ANUIES, vol. XXXVII (2) nº 146 abril-junio, pp. 11-40.

Reynaga Obregón S. (coordinadora) (2003), Educación, trabajo, ciencia y tecnología. Consejo Mexicano de Investigación Educativa. Serie "La investigación educativa en México, 1992-2002”, vol. 6.

Rubio Oca J. (coord.) (2006), La política educativa y la educación superior en México.1995-2006. Un balance. México. Fondo de Cultura Económica/ Secretaría de Educación Pública. 317 p. 
Secretaría de Educación Pública (2008), Sistema Educativo de los Estados Unidos Mexicanos. Principales cifras. Ciclo escolar 2006-2007, SEP, México, SEP. Estadística Histórica. Sistema para el análisis de la estadística educativa http://www.sep.gob.mx/ work/appsite/nacional/index.htm

Solana F. (1982), Historia de la educación pública en México, Tomo II, México, FCE, 1982.

Suárez Zozaya M.H. (2005), Jóvenes mexicanos en la feria del mercado de trabajo. Conveniencias e inconveniencias de tener educación superior. México, UNAM/Miguel Ángel Porrúa, 66 p.
Tokman V. (2004), "De la informalidad a la modernidad” Formación en la economía informal. Boletín Cinterfor (Montevideo) (155), pp. 9- 31.

Vargas R (2006), Perfiles de competencias profesionales demandadas por cinco sectores productivos en la ciudad de Tijuana. Instituto Tecnológico de Tijuana, México. Ediciones del XXXV aniversario.

Vega Tato G (à paraître), Instituciones particulares emergentes de Educación superior. Entre la demanda, la calidad percibida y la satisfacción estudiantil. Tesis de doctorado en proceso. DIE Cinvestav IPN México.

\section{Résumé}

Priorité à la formation scolaire pour le travail au Mexique.

Tensions et contradictions entre l'État, les secteurs professionnels et les étudiants

Maria de lbarrola

Cet article est un essai sur la primauté accordée au Mexique à la formation scolaire pour le travail. II analyse la façon dont le gouvernement fédéral a assumé cette responsabilité et le type de tensions et de contradictions entre les propositions gouvernementales, la vision sommaire des secteurs professionnels, les possibilités des institutions scolaires créées et les demandes et aspirations des jeunes. La méconnaissance de l'hétérogénéité des marchés du travail du pays constitue un facteur explicatif des logiques et rationalités qui fondent les divers intérêts de chacun des acteurs et qui s'expriment dans la structure actuelle de la formation.

Mots clés

Formation professionnelle, enseignement supérieur, secteur économique, Mexique

Journal of Economic Literature: I 21 